\title{
SEBACEOUS CARCINOMA - UNEXPECTED TUMOR OF THE NASAL ALA DORSUM
}

\author{
At. Vlaykov', At. Stefanov', V. Velev' ${ }^{2}$, M. Gulubova², J. Ananiev ${ }^{2}$ \\ ${ }^{1}$ Department of Otorhinolaryngology, Trakia University, Medical Faculty - Stara Zagora, Bulgaria \\ ${ }^{2}$ Department of Clinical Pathology, Trakia University, Medical Faculty - Stara Zagora, Bulgaria
}

\begin{abstract}
Today, the extraocular variant of sebaceous carcinoma is still being poorly recognized. This type of carcinoma is rarely diagnosed correctly, which, together with its aggressive behavior, contributes to its poor prognosis. We present a case of an 84-year-old man with a history of left nasal ala tumor formation, diagnosed morphologically and immunohistochemically as sebaceous carcinoma.
\end{abstract}

Key words: sebaceous carcinoma, nasal dorsum, immunohistochemistry

Corresponding author: Atanas Vlaykov, MD, e-mail: at. vlaykov@gmail.com

\section{INTRODUCTION}

or the first time sebaceous carcinoma (SC) was described by Srtraatsma in 1950s. Nowadays, this adnexal malignant neoplasm that we know of for decades could be divided into two types: the most often type, the one found in the periocular area - an ocular variant, and the most rare type - the extraocular variant $[1,2]$.

Sometimes the tumor could appear as a diagnostic sign of Muir-Torre syndrome - a disorder, associated with visceral malignancies and gene abnormalities. Patients must be carefully evaluated for a family history of cancer, therefore physical examination should be thoughtfully performed. [3]. As it is the case with periocular SC, extraocular carcinoma could also be an aggressive malignancy, with a variety of reports on the incidence of metastasis and mortality [4].

The classical characteristics of SC are a tumor with asymmetrical and infiltrative borders, a one that includes irregular aggregates of neoplastic sebocytes with variation in size and shape, atypical cells with pleomorphic nuclei and a different degree of sebaceous differentiation. The tumor has specific signs, but sometimes in certain circumstances, the use of immunohistochemistry may facilitate the identification of the specific diagnosis.

Here is being presented a case of a patient with nasal ala tumor formation with estimated progressive growth. After total excision and histopathological examination, the diagnosis was confirmed as being sebaceous carcinoma of the left nasal ala.

\section{CASE}

An 84-year-old male patient was introduced to the ENT Clinic in University Hospital, Stara Zagora. He presented with a 4-year history of left nasal ala tumor formation. The mass has progresisvly increased and the tumor has grown quickly during the last few months. There has not been spontaneous or provoked pain. Recently, there have been cases of spontaneous bleedings of the formation after mechanical stimulations. Physical examination demonstrated a 
well defined $3 \times 3 \mathrm{~cm}$ soft skin-colored nodule with uneven surface, located on a broad basis of the left nasal wing. There were some reddish spots over the mass without ulceration. Cranial and cervical lymph nodes were not palpable. The blood tests did not show any changes.

The formation was totally excised and sent to a laboratory for pathohistological analysis where the defect was resurfaced with a full-thickness skin graft from the cervical region.

After dehospitalisation, the patient was diverted toward Regional Oncologic Center for observation, and possibly for radiotherapy.

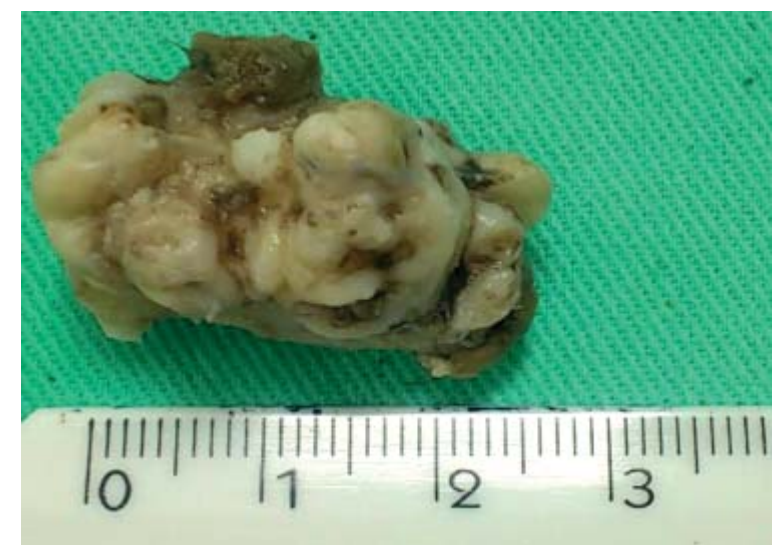

Fig. 1. Gross view of tumour mass - soft skin-colored nodule with uneven surface

\section{Histology}

Unexpectedly, the histological examination of the biopsy revealed a diagnosis of sebaceous carcinoma. The lesion with infiltrative borders was composed of aggregates varying in sizes and shapes, bearing little to normal resemblance to sebaceous lobules; clusters of clear cells with vacuolated cytoplasm with hyperchromatic irregular nuclei and numerous and abnormal mitotic figures. Between some shapes were seen foci of necrosis (Fig. 2a-c). Immunohistochemical analysis demonstrated that the tumor cells were strongly positive for Cytokeratin, weakly positive for epithelial membrane antigen (EMA), but negative for S100 (Fig. 2d-f).

Other sebaceous lesions such as basal cell carcinoma, Merkel cell carcinoma, etc., were ruled out as part of the differential diagnosis.

\section{DISCUSSION}

According to recent research, SC most frequently occurs in the periorbital area and accounts for $2-7 \%$ of all malignant eyelid tumors, while the extraocular type accounts for approximately $25 \%$ of all SCs, affecting the head and the neck, and less common the trunk, genitalia and extremities [5, 6]. Clinically this type of tumor has no pathognomonic characteristics that could make it differentiable from other skin le-

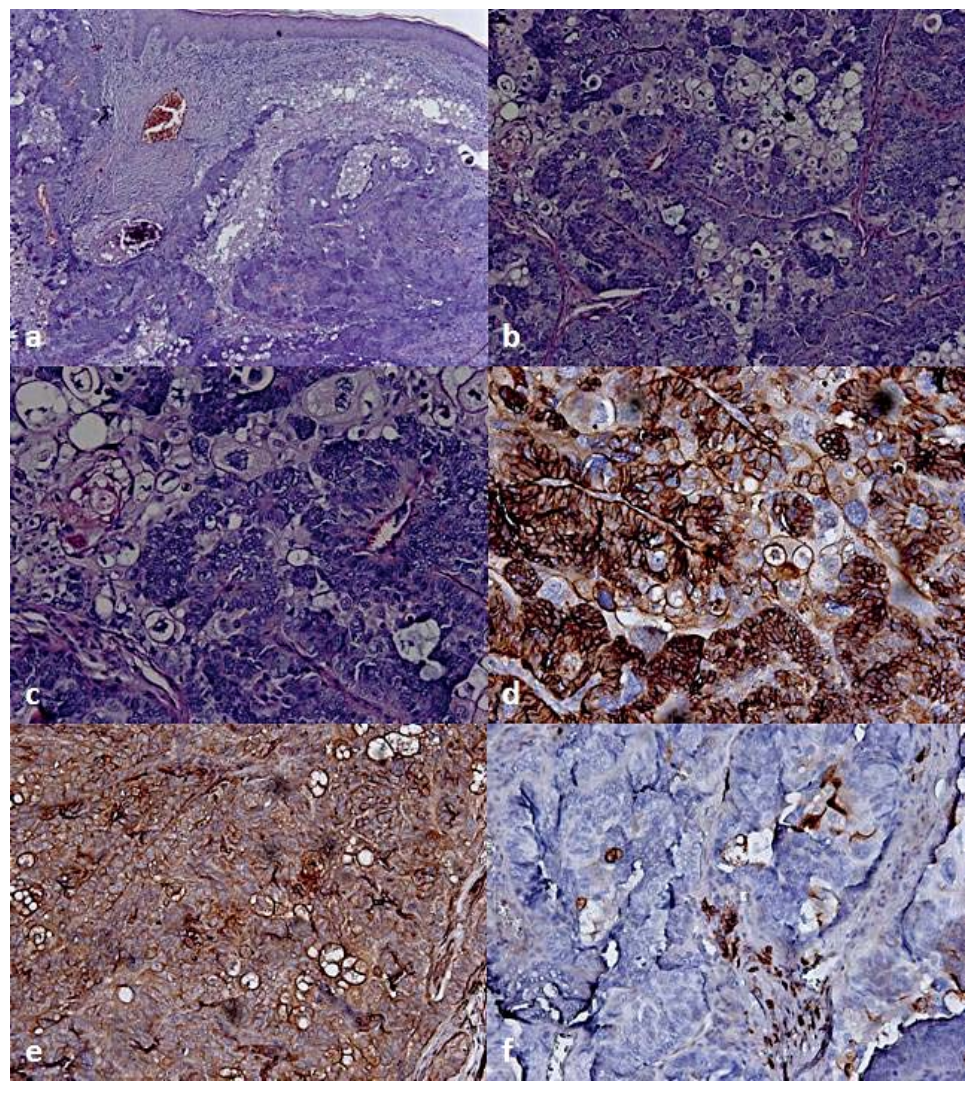

Fig. 2. Tumour composed from clear cells with vacuolated cytoplasm with hyperchromatic irregular nuclei and numerous and abnormal mitotic figures and magnifications a) (x50); b) (x200); c) (x200); d) strong expression of Cytokeratin (x200) e) strong/moderate expression of EMA (x200); f) lack of expression of S100 in tumour cells (x200) 
sions in the same area, resulting in the frequent need for it to be carefully distinguished from other benign or malignant skin lesions $[6,7]$.

In most cases, tumor cells could be described as cells with hyperchromatic nuclei of variable sizes, mitotic activity and basaloid characteristics, having three grades of differentiation: well, moderately, or poorly differentiated tumor. In some cases, welldifferentiated SCs are expected to be distinguished from sebaceous adenomas by their infiltrative growth pattern, deep extension, and different stage of atypia, as in our case.

Histochemically, tumors could be stained with fat stain reactive - Oil Red and Sudan. Immunohistochemically the tumors stain positively for cytokeratin, anti-epithelial membrane antigen (EMA) and LeuM1, and stain negatively for carcinoembryonic antigen (CEA) and S100.

The differential diagnoses of SC should include other melanocytic and non-melanocytic lesions, such as malignant melanoma, squamous cell carcinoma, basal cell carcinoma and Merkel cell carcinoma.

Interest can represent the association of SC with the so-called Muir-Torre syndrome. The latter is characterized by the association of some sebaceous tumors (incl. SC), with or without a keratoacanthoma, with one or more visceral neoplasms such as tumors arising from the gastrointestinal tract, the proximal colon, followed by tumors of the genitourinary tract [8].

Last but not least, targeted assessment for regional or distant metastases is an important step in the diagnostic work-up. Some authors confirm that head and neck localization of SC with regional nodal involvement is not uncommon and the rate of regional nodal involvement is $10-15 \%[9,10]$. Almost similar data is reported by other authors as well. They conclude that recurrence is observed in no more than $1 / 3$ of the patients, and metastasis could be detected in regional and distant lymph nodes [11, 12].

There is insufficient information in the literature about the therapeutic behavior and prognosis of SC with such location. In relation to our specific patient, we chose to perform a local excision without further lymph dissection. During the follow-up period neither recurrence, nor metastases were established. Scarce literature suggests that excision is quite sufficient and there is no correlation between the therapeutic effect and the more aggressive course of the disease [13]. There is also no evidence of any differences between usage of Mohs micrographic surgery compared to the simple local excision in terms of progression and cosmetic effect [14].
In conclusion, we present a rare case of sebaceous carcinoma located on the nasal wing, which lacked characteristic clinical manifestation. The presence of specific morphological and immunohistochemical findings favored the diagnosis of sebaceous carcinoma without evidence of close and distant metastases.

\section{Conflict of interests}

The authors have nothing to disclose.

\section{REFERENCES}

1. Nelson BR, Hamlet KR, Gillard M et al. Sebaceous carcinoma. J Am Acad Dermatol. 1995;33:1-15.

2. Bongu A, Lee ES, Peters SR, Chokshi RJ. Locally aggressive and multicentric recurrent extraocular sebaceous carcinoma: case report and literature review. Eplasty. 2013;21;13:e44.

3. Cieza-Díaz DE, Cano-Martínez N, Barchino-Ortiz L, LongoImedio I. Extraocular sebaceous carcinoma: a report of 2 cases. Actas Dermosifiliogr. 2012;103(10):919-22.

4. Shalin SC, Lyle S, Calonje E, Lazar AJ. Sebaceous neoplasia and the Muir-Torre syndrome: important connections with clinical implications. Histopathology. 2010;56(1):133-47.

5. Rao NA, Hidayat AA, McLean IW, Zimmerman LE. Sebaceous carcinomas of the ocular adnexa: A clinicopathologic study of 104 cases, with five-year follow-up data. Hum Pathol. $1982 ; 13(2): 113-22$.

6. Dummer R, Pittelkow MR, Iwatsuki K et al. Skin Cancer - A World-Wide Perspective. Springer 2011. 153-154.

7. Shields JA, Shields CL. Eyelid, Conjunctival and Orbital Tumours: atlas and textbook 2nd ed. Lippincott W\&W 2008. 50-52.

8. Cieza-Díaz DE, Cano-Martínez N, Barchino-Ortiz L, LongoImedio I. Extraocular sebaceous carcinoma: a report of 2 cases. Actas Dermosifiliogr. 2012;103(10):919-22.

9. Nijhawan N, Ross MI, Diba R et al. Experience with sentinel lymph node biopsy for eyelid and conjunctival malignancies at a cancer center. Ophthal Plast Reconstr Surg 2004; 20: 291-295.

10. Osada $\mathrm{S}$, Ueno $\mathrm{T}$, Inai $\mathrm{S}$ et al. Sebaceous carcinoma of the nose with a regional metastasis following false-negative sentinel lymph node biopsy. Acta Derm Venereol. 2011;91(3): 367-8.

11. Alawi F, Siddiqui A. Sebaceous carcinoma of the oral mucosa: Case report and review of the literature. Oral Surg Oral Med Oral Pathol Oral Radiol Endod 2005; 99: 79.

12. Hornblass A, Lauer SA. Sebaceous carcinoma of the eyelids. Ophthalmology 2004; 111: 2149.

13. Wick M, Swanson PE. Cutaneous adnexal tumors: a guide to pathologic diagnosis. Am Soc Clin Pathol. 1991;113:374-382.

14. Phan K, Loya A. Mohs micrographic surgery versus wide local excision for sebaceous adenocarcinoma of the eyelid: Analysis of a national database. J Plast Reconstr Aesthet Surg. 2019 Mar 2. pii: S1748-6815(19)30074-9.

Received: Febr, 2019 - Revised: April, 2019 Accepted: June, 2019 